$\begin{array}{llllllllllll}\text { A C T A } & \text { C H E M I C A } & \text { S C A N D I N A V I C A } & 27 & \text { (1973) } & 915-923\end{array}$

\title{
A Dependence of Transition Properties of the Univalent Nitrates upon Structural Entities
}

\author{
J. H. F ERMOR and A. KJ EKSHUS \\ Kjemisk Institutt, Universitetet i Oslo, Blindern, Oslo 3, Norway
}

\begin{abstract}
Low temperature dielectric transitions occurring in $\mathrm{LiNO}_{3}, \mathrm{NaNO}_{3}$, $\mathrm{KNO}_{3}, \mathrm{RbNO}_{3}, \mathrm{CsNO}_{3}, \mathrm{AgNO}_{3}, \mathrm{TlNO}_{3}$, and $\mathrm{NH}_{4} \mathrm{NO}_{3}$ are shown to have characteristic temperatures which depend on the degree of packing of ions in the crystal lattice. The same factor is also shown to influence the melting points of the compounds. As an example of transformations at intermediate temperatures, observed values of dielectric constant in $\mathrm{KNO}_{3}$ at $1 \mathrm{kHz}$ are considered in relation to the phase II to I transition at $\sim 402 \mathrm{~K}$. The range of existence of phase IIIm ( $\mathrm{m}=$ metastable) appears to be connected with a region of increased dielectric constant in phase II.
\end{abstract}

Changes in the physical properties of univalent nitrates with temperature

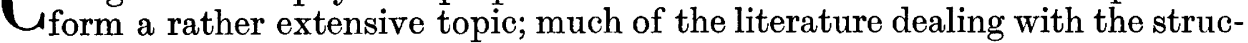
tural and order-disorder transformations occurring above room temperature. The present work is concerned primarily with the low temperature, and melting transitions, since these are found to have features in common. Between these temperature extremes, the phase II to I transformation in $\mathrm{KNO}_{3}$ is taken as an example of a first order structural transition.

\section{TRANSFORMATIONS BELOW ROOM TEMPERATURE}

As reported earlier, ${ }^{1}$ dielectric transitions occur below room temperature in $\mathrm{LiNO}_{3}(263 \mathrm{~K}), \mathrm{NaNO}_{3}(243 \mathrm{~K}), \mathrm{KNO}_{3}(213 \mathrm{~K}), \mathrm{RbNO}_{3}(228 \mathrm{~K}), \mathrm{CsNO}_{3}(238 \mathrm{~K})$, $\mathrm{AgNO}_{3}(238 \mathrm{~K}), \mathrm{TlNO}_{3}(238 \mathrm{~K})$, and $\mathrm{NH}_{4} \mathrm{NO}_{3}(241 \mathrm{~K})$; the temperatures in parentheses being characteristic of the transitions on cooling in each case. In general, the transitions are detected by a peak in the dielectric constant $\epsilon$ on cooling, with a discontinuity in slope at the maximum value. On heating, there is a considerably larger and more rounded maximum at a somewhat higher temperature. Corresponding minima are found in the a.c. resistivities. In the case of $\mathrm{KNO}_{3}$, a determination of unit cell dimensions showed there to be an anomalous variation in cell volume in the region of the transition. ${ }^{2}$ That lattice expansion can have an important bearing on dielectric transitions has been shown on

Acta Chem. Scand. 27 (1973) No. 3 
theoretical grounds by Simpson ${ }^{3}$ for cases where there is a change in the freedom of orientation of electric dipoles.

The transitions in the univalent nitrates appear to involve only small changes in energy, since no corresponding anomalies are noted in the specific heat curves of $\mathrm{NaNO}_{3}$ and $\mathrm{KNO}_{3},{ }^{4}$ or by means of differential thermal analysis in $\mathrm{RbNO}_{3}$ and $\mathrm{CsNO}_{3} \cdot{ }^{5}$ In $\mathrm{TINO}_{3}$, on the other hand, a discontinuity in slope has been noted ${ }^{6}$ in previously published specific heat data.

The dielectric anomalies are in themselves insufficient to enable the nature of the transitions to be determined in detail, but some progress in interpretation may be made, pending the use of supplementary experimental techniques. The values of the electrical parameters obtained below room temperature for $\mathrm{LiNO}_{3},{ }^{7} \mathrm{NaNO}_{3},{ }^{8} \mathrm{KNO}_{3},{ }^{2} \mathrm{RbNO}_{3},{ }^{9} \mathrm{CsNO}_{3},{ }^{9} \mathrm{AgNO}_{3},{ }^{6} \mathrm{TINO}_{3},{ }^{6}$ and $\mathrm{NH}_{4} \mathrm{NO}_{3}{ }^{6}$ suggest that the anomalies reflect considerable alterations in lattice forces. The form of the curves does not, for example, appear to be consistent with an impurity relaxation effect, or to result from the reorientation of dipoles constituted by interstitial ions closely associated with vacancies in the corresponding sublattice. These phenomena would be expected to result in more symmetrical maxima and minima, smaller variations in $\epsilon$, and an absence of hysteresis, as may be seen for example in Ref. 10.

Although the exclusion of water from the nitrate samples was given a high priority, at the cost of obtaining non-standard values of dielectric parameters, the complete absence of water is unlikely. The dielectric transitions do not reflect a freezing and remelting of solutions of the salts, however, the eutectic temperatures being in most cases much higher. If additional impurities were included in solution, thus reducing the operative eutectic temperatures, the anomalous increases in dielectric constant on heating which were found for $\mathrm{KNO}_{3}(\Delta \epsilon=9.2), \mathrm{RbNO}_{3}(\Delta \epsilon=2.5), \mathrm{CsNO}_{3}(\Delta \epsilon=4.0)$, and $\mathrm{AgNO}_{3}(\Delta \epsilon=2.8)$ would indicate extremely high impurity contents, and therefore this cannot be regarded as a reasonable explanation of the anomalies.

The dielectric anomalies may, however, reflect structural or order-disorder transformations; and it is natural to suppose that the nitrate groups play an important role by reason of internal changes, modifications in ordering, or a combination of such effects. A noticeable feature of the transitions is that on heating the samples, an increasing value of $\epsilon$ is continued into the region of the normal room temperature phase to form a maximum. Such high values of $\epsilon$ as those recorded, in combination with low a.c. resistivities, can be most simply accounted for by the rotation of electric dipoles through restricted angles, by large ionic displacements, or a combination of the two. Dipoles could, for example, arise from distortions from planarity of the nitrate groups, and the transitions involve the ordering of these dipoles. The symmetrical out of plane mode of oscillation of the group, of frequency $v_{2} \approx 830 \mathrm{~cm}^{-1}$, is largely restricted to the ground state within the temperature range of the anomalies ( 210 to $265 \mathrm{~K}$ ), and this favours a locking of distorted groups into an ordered structure.

The possibility that the dielectric transitions reflect structural orderdisorder transformations is supported by the similarity of the experimental data with those obtained by Guillien ${ }^{12}$ for the lambda transitions in ammonium halides at comparable temperatures. The first of these transformations was 
discovered calorimetrically by Simon ${ }^{13}$ in $\mathrm{NH}_{4} \mathrm{Cl}$, and the results of a large number of investigations including those using neutron diffraction ${ }^{14,15}$ show that whereas the orientations of the $\mathrm{NH}_{4}^{+}$tetrahedra are ordered in the low temperature phases, at room temperature they are randomly distributed between two alternative orientations. Some aspects of the orderdisorder process itself are still uncertain, however, in spite of the large number of studies on the subject. By analogy, it may be suggested as a possibility that the low temperature dielectric transitions in the univalent nitrates correspond to the onset of orientational disorder of the nitrate group. Only very small amounts of energy are involved in reorientations between equivalent positions at low frequencies. If the nitrate group is regarded as planar, as claimed by Walsh ${ }^{16}$ on quantum mechanical grounds, the most probable reorientations are those through angles of $120^{\circ}$ about the threefold axis of the group. These reorientations would be consistent with the suggestion by Schroeder et al., ${ }^{17}$ on the basis of infrared measurements, that the barrier opposing reorientation is surmounted at room temperature in several nitrates.

An additional possibility which cannot be definitely excluded at present, however, is that small departures from planarity of the nitrate groups are involved in the transitions. In this case, additional crystallographic parameters are introduced in the structural model, with corresponding possibilities for disordering. Whichever form the disorder takes, it is interesting to consider the variation of the transition temperature $T_{a}$ within the family of compounds. It is reasonable to suppose that anion-cation and anion-anion interactions influence this parameter, and therefore the lattice volume $V_{l}$ associated with each anion is selected as an independent variable. Thus $V_{l}$ is the unit cell volume per formula unit less that of the cation. The latter is assumed to be spherical, and of radius $r_{c}$ appropriate to sixfold coordination.

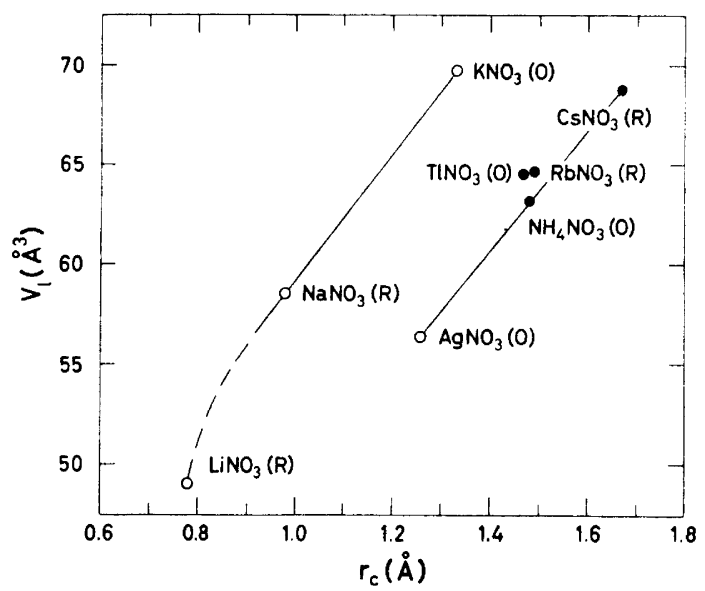

Fig. 1. Lattice volume per anion $V_{l}$ versus cation radius $r_{c}$ for univalent nitrates. The crystal structures of the room temperature phases are (R) rhombohedral, and (O) orthorhombic. Filled circles represent compounds adopting a cubic phase I structure $\left(r_{c}>1.4 \AA\right)$.

Acta Chem. Scand. 27 (1973) No. 3 


\section{INFLUENCE OF CATION RADIUS UPON PACKING}

Before examining the dependence of $T_{a}$ upon $V_{l}$, it is interesting to see from Fig. 1 that the connection between $V_{l}$ and $r_{c}$ is such that the compounds fall into two, approximately linear groups. The values of $r_{c}$ employed in this diagram may be regarded as having been well established, except possibly in the case of $\mathrm{Li}^{+}$, for which Goldschmidt's value of $0.78 \AA^{18}$ has been selected, consistent with its use in related work. ${ }^{6}$ The values of $V_{l}$ are calculated for the room temperature phase in each case. There is seen to be a degree of correlation between the data for $\mathrm{LiNO}_{3},{ }^{19} \mathrm{NaNO}_{3},{ }^{20}$ and $\mathrm{KNO}_{3},{ }^{21}$ despite differences in structure, which may be obtained from the references given. The overall symmetries only are indicated in the figure, like symbols not necessarily indicating like structures. The data for $\mathrm{RbNO}_{3},{ }^{22} \mathrm{CsNO}_{3},{ }^{23} \mathrm{TlNO}_{3},{ }^{24}$ and $\mathrm{NH}_{4} \mathrm{NO}_{3},{ }^{25}$ form a separate group, the members of which have cubic high temperature phase I structures, ${ }^{26}$ while $\mathrm{LiNO}_{3}, \mathrm{NaNO}_{3}, \mathrm{KNO}_{3}$, and $\mathrm{AgNO}_{3}$ are rhombohedral, at least in their high temperature phases. ${ }^{27}$ Thus, independent of the structure type at room temperature, that of the highest temperature phase depends on whether $r_{c} \gtrless 1.4 \AA$. The separation of the room temperature data of Fig. 1 into two linear sets suggests two types of lattice bonding, independent of the crystal structures involved.

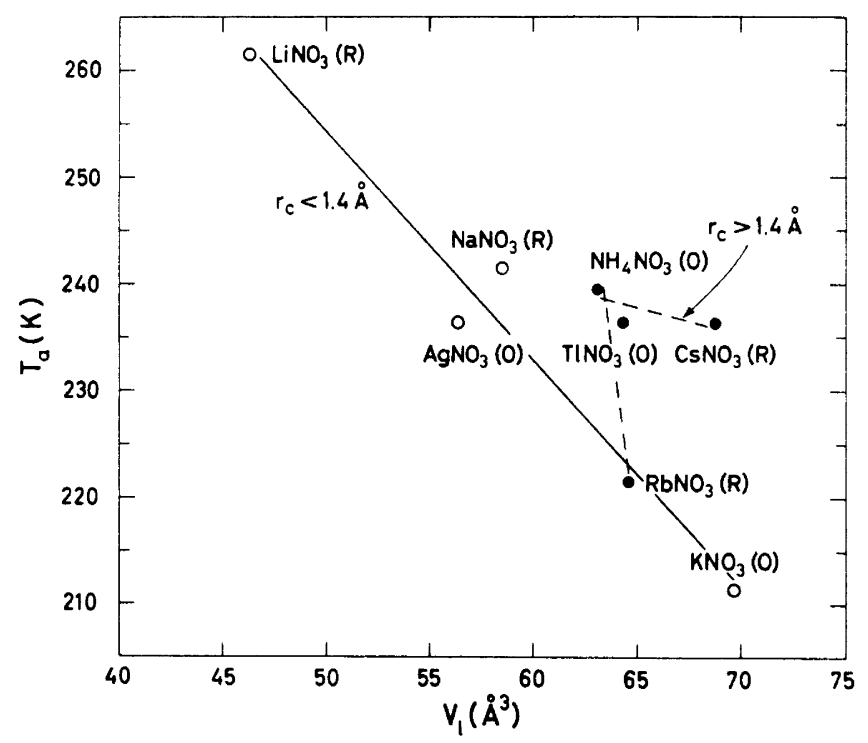

Fig. 2. The low temperature dielectric transition temperatures $T_{a}$ versus the lattice volume per anion $V_{l}$ for univalent nitrates. The crystal structures of the room temperature phases are $(\mathrm{R})$ rhombohedral and $(\mathrm{O})$ orthorhombic. Filled circles denote compounds with $r_{c}>1.4 \AA$. 


\section{DEPENDENCE OF DIELECTRIC TRANSITION TEMPERATURE ON PACKING}

No consistent trend including all of the compounds is to be found on plotting $T_{a}$ versus $r_{c}$, but overall correlations between $T_{a}$ and $V_{l}$ may be seen in Fig. 2. The results suggest that in general the lesser the ionic interaction (i.e. the greater $V_{l}$ ), the lower the transition temperature. As in Fig. 1, the influence of cation radius also appears to be in evidence in Fig. 2, since the points fall into two groups according to whether $r_{c} \gtrless 1.4 \AA$. In order of increasing $r_{c}$, the group comprising $\mathrm{LiNO}_{3}, \mathrm{NaNO}_{3}, \mathrm{AgNO}_{3}$, and $\mathrm{KNO}_{3}$ is approximately linear, while $\mathrm{TlNO}_{3}, \mathrm{NH}_{4} \mathrm{NO}_{3}, \mathrm{RbNO}_{3}$, and $\mathrm{CsNO}_{3}$ may also form a related group if $\mathrm{RbNO}_{3}$ or $\mathrm{CsNO}_{3}$ are excepted. It should be noted that the probable error limits in the values of $T_{a}$ are not well defined, but may provisionally be set at $\pm 5 \mathrm{~K}$. It may be relevant that $\mathrm{RbNO}_{3}$ is unusual among these compounds in undergoing a small contraction on melting, ${ }^{28}$ suggesting peculiar bonding properties. The indicated correlations, which imply $T_{a}$ to be a monotonic function of $V_{l}$ for each group of compounds, are of course to be regarded as suggestive rather than conclusive. It is not supposed that proximity effects alone determine the value of $T_{a}$, but the results do tend to confirm the fundamental or intrinsic nature of the anomalies, and may assist in the choice of methods suitable for their further investigation. The correlations are consistent with the order-disorder models considered above in selecting $V_{l}$ as independent parameter.

The influence of packing upon the internal characteristics of nitrate groups has been demonstrated by Bues, ${ }^{29}$ who showed a dependence of the symmetrical in-plane oscillation frequency $v_{1}$ upon the free lattice volume per anion $V_{f}$ (i.e. $V_{f}=V_{l}-V_{a}$, where $V_{a}$ is the volume of the anion). Bues considered $\mathrm{LiNO}_{3}, \mathrm{NaNO}_{3}, \mathrm{KNO}_{3}$, and $\mathrm{AgNO}_{3}$, in solid and liquid states; finding no evidence of variation in the strength of the internal binding forces of the nitrate groups in these compounds, with the exception of $\mathrm{AgNO}_{3}$.

\section{INFLUENCE OF PACKING ON THE MELTING TRANSFORMATION}

It was proposed earlier ${ }^{30}$ that the thermal response of the anion sublattice is primarily responsible for the anomalously low melting points $T_{f}$ of the univalent nitrates, whose values are some 200 to $400 \mathrm{~K}$ lower than for the alkali halides. The relationship between $T_{\text {, and }} V_{l}$ for the univalent nitrates is shown in Fig. 3, where it is seen that, with the exception of $\mathrm{CsNO}_{3}$, the alkali metal nitrates show an approximately linear correlation. It is important to note that $\mathrm{KNO}_{3}$ provides an exception to the ordering of points according to cation radius. The influence of ionic radius upon $T$, for the alkali halides, which provide a convenient class of compounds for comparison, has been noted earlier. ${ }^{30}$ The deviation of $\mathrm{CsNO}_{3}$ may perhaps be due to its limiting value of cation radius, possibly resulting from a screening effect. The influence of $V_{l}$ upon $T_{t}$ for the alkali metal nitrates is consistent with both a variation in lattice energy and a modification of the nitrate groups, on the assumption that the modes of oscillation or disorder of the latter, over and above those of spherical ions, control the melting process.

Acta Chem. Scand. 27 (1973) No. 3 


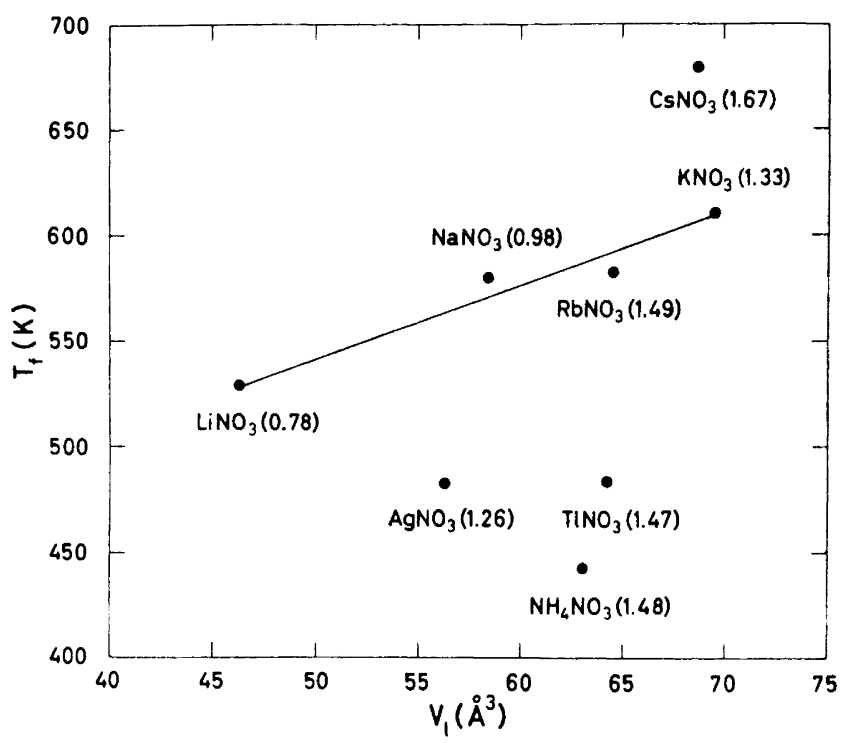

Fig. 3. Melting points $T_{f}$ versus lattice volume per anion $V_{l}$ for univalent nitrates. Values of cation radii are shown in parentheses.

The positions of $\mathrm{AgNO}_{3}, \mathrm{TlNO}_{3}$, and $\mathrm{NH}_{4} \mathrm{NO}_{3}$, relative to the other compounds in Fig. 3, clearly result from the operation of factors additional to those considered above. The electronic polarizabilities of the ions might be one such factor, but while the value for $\mathrm{Tl}^{+}\left(90.1 \AA^{3}\right)$ exceeds even that of $\mathrm{Cs}^{+}\left(71.3 \AA^{3}\right)$, that for $\mathrm{Ag}^{+}\left(39.4 \AA^{3}\right)$ is less than for $\mathrm{K}^{+}\left(47.3 \AA^{3}\right)$, according to the data reported by Roberts. ${ }^{31}$ Additional evidence for the uniformity of anion-cation bonding in univalent nitrates is presented elsewhere. ${ }^{6}$

In regarding ionic interactions as the controlling factor in determining $T$, for $\mathrm{LiNO}_{3}, \mathrm{NaNO}_{3}, \mathrm{KNO}_{3}, \mathrm{RbNO}_{3}$, and $\mathrm{CsNO}_{3}$, variations in the internal mode frequencies of the anion have not been mentioned. According to the concept of the "Lockerungseffekt" introduced by Theimer, ${ }^{32}$ however, deformation of the anion by the electrostatic field generated between it and the surrounding cations can diminish the internal binding forces of the anion, with a consequent reduction in internal mode frequencies. This has been shown by Bues ${ }^{29}$ to occur in $\mathrm{AgNO}_{3}$, but not in $\mathrm{LiNO}_{3}, \mathrm{NaNO}_{3}$, and $\mathrm{KNO}_{3}$, and it is possible therefore that the low value of $T_{t}$ for $\mathrm{AgNO}_{3}$, and probably also for $\mathrm{TlNO}_{3}$, reflects a displacement to lower temperatures of the activation of internal modes.

Also important in an understanding of the melting process is the exceptionally high self diffusion rate of cations in the vicinity of the melting point, which has been clearly shown to exist in the high temperature disordered state of $\mathrm{NaNO}_{3}$ by means of $\mathrm{NMR},{ }^{33},{ }^{34}$ d.c. conductivity, ${ }^{8}$ and low frequency dispersion ${ }^{35}$ experiments. These results show an instability of the cation sublattice which makes cooperative anion vibrations particularly effective in producing the disorder of melting. 
In this connection, values of the partition functions for simple harmonic oscillators of the frequencies of internal oscillation of the nitrate group show that appreciable activation occurs in the temperature range of the thermal transformations above room temperature, and of the melting points. ${ }^{36}$ These vibrations tend to increase the effective degree of sphericity of the group, and to cause fluctuations in the lattice barrier potentials opposing positional and orientational disorder of the ions. On increasing the temperature of a crystal, both of the above consequences of vibration tend to favour transformations into structures of a higher degree of symmetry and disorder, and lead eventually to the melt.

\section{THE FIRST ORDER STRUCTURAL TRANSFORMATION IN $\mathrm{KNO}_{3}$}

The most common type of solid-state transformation occurring in the univalent nitrates is the discontinuous, or first order structural transition. As an example of this type of transformation, that between phases II and $\mathrm{I}$ in $\mathrm{KNO}_{3}$ at $\sim 402 \mathrm{~K}$ is extremely sharp, as may be seen from the temperature variation of the electrical resistivity. ${ }^{37}$ If the characteristic modes of anion vibration are of importance in causing this and other transformations in the nitrates, the sharpness of the transition seems paradoxical in view of the gradual thermal activation of the vibrational modes. Experimental evidence of changes premonitory to the II to I transformation in $\mathrm{KNO}_{3}$ is, however, shown in Fig. 4, where there is seen to be a gradual increase in the dielectric constant, beginning at $\sim 350 \mathrm{~K}$. These data were obtained for a polycrystalline p.a. grade (Merck) sample, according to the method described in Ref. 8. The measuring frequency used was $1 \mathrm{kHz}$, compared with the higher values $(\geqq 10 \mathrm{kHz})$ employed in previous determinations, ${ }^{38-42}$ with which the present results are consistent. No other premon-

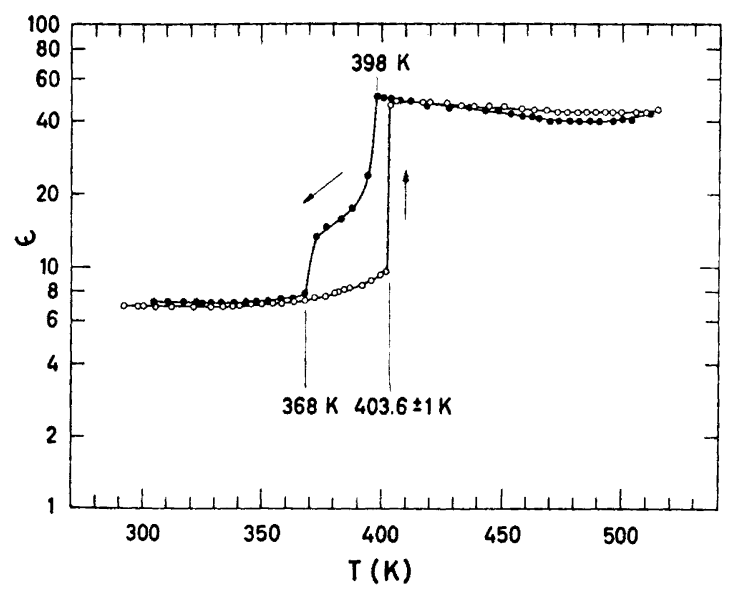

Fig. 4. Dielectric constant $\in$ versus absolute temperature $T$ for polycrystalline p.a. grade $\mathrm{KNO}_{3}$ at $1 \mathrm{kHz}$. Arrows indicate the direction of temperature change.

Acta Chem. Scand. 27 (1973) No. 3 
itory effects are known to have been found in connection with this transformation. Also to be seen in the diagram is a high value of $\epsilon$ of $\sim 50$ in phase I $(T>403.6 \mathrm{~K})$, where there is known to be orientational disorder of the nitrate groups, ${ }^{43}$ leading to a reduction in mean energy barriers. The formation of the intermediate phase $\operatorname{IIIm}(\mathrm{m}=$ metastable; $368<T<398 \mathrm{~K}$, approximately) on cooling is also apparent.

The observed increase in $\epsilon$ prior to the phase II to I transformation is also relevant to the soft mode interpretation of phase transitions. According to this model, the lattice modes directly associated with the displacement of atoms to new structural positions become unstable as the transition is approached. Thus, diminished values of force constants cause the frequencies of certain lattice modes to fall to zero at the transition temperature; and the reduced force constants result in increased displacements in the presence of an externally applied electric field, thus causing the observed increase in $\epsilon$. The stability of the phase IIIm on cooling is no doubt related to the increased value of $\epsilon$ of phase II over the temperature range of existence of the phase IIIm recorded here. The transitional quality of phase IIIm is apparent from its values of $\epsilon$.

Treatments of internal and librational modes of oscillation of molecules or ionic groups on the one hand, and of soft lattice modes on the other, provide complementary aspects of transformations in crystals. Whereas soft mode studies lead to insight into the transitional mechanism connecting initial and final lattice structures, consideration of spatial factors, molecular or ionic group disorder, and of internal modes and their interactions have been found of value in suggesting how solid state and melting transformations originate in the compounds considered.

Acknowledgements. This work was made possible by the financial support of Norges almenvitenskapelige forskningsråd.

\section{REFERENCES}

1. Fermor, J. H. and Kjekshus, A. Acta Chem. Scand. 22 (1968) 2054.

2. Fermor, J. H. and Kjekshus, A. Acta Chem. Scand. 22 (1968) 836.

3. Simpson, J. H. Can. J. Phys. 29 (1951) 163.

4. Southard, J. C. and Nelson, R. A. J. Am. Chem. Soc. 55 (1933) 4865.

5. Owen, R. W. and Kennard, C. H. L. Aust. J. Chem. 24 (1971) 1295.

6. Fermor, J. H. and Kjekshus, A. To be published.

7. Fermor, J. H. and Kjekshus, A. Acta Chem. Scand. 23 (1969) 1581.

8. Fermor, J. H. and Kjekshus, A. Acta Chem. Scand. 22 (1968) 1628.

9. Fermor, J. H. and Kjekshus, A. Acta Chem. Scand. 26 (1972) 2645.

10. Breckenridge, R. G. J. Chem. Phys. 18 (1950) 913.

11. Herzberg, G. Infrared and Raman Spectra of Polyatomic Molecules, Van Nostrand, New York 1945.

12. Guillien, R. Compt. Rend. 208 (1939) 1561.

13. Simon, F. Ann. Physik 68 (1922) 241.

14. Levy, H. A. and Peterson, S. W. J. Am. Chem. Soc. 75 (1953) 1536.

15. Goldschmidt, G. H. and Hurst, G. D. Phys. Rev. 86 (1952) 797.

16. Walsh, A. D. J. Chem. Soc. 19532301.

17. Schroeder, R. A., Weir, C. E. and Lippincott, E. R. J. Res. Natl. Bur. Std. A 66 (1962) 407. 
18. Goldschmidt, V. M. Skrifter Norske Videnskaps-Akad. Oslo, I: Mat.-Naturv. Kl. 1926. No. 1 .

19. Zachariasen, W. H. Skrifter Norske Videnskaps-Akad. Oslo, I: Mat.-Naturv. Kl. 1928 No. 4.

20. Cherin, P., Hamilton, W. C. and Post, B. Acta Cryst. 23 (1967) 455.

21. Edwards, D. A. Z. Krist. 80 (1931) 154.

22. Brown, R. N. and McLaren, A. C. Acta Cryst. 15 (1962) 974.

23. Delacy, T. P. and Kennard, C. H. L. Aust. J. Chem. 24 (1971) 165.

24. Brown, R. N. and McLaren, A. C. Acta Cryst. 15 (1962) 977. .

25. West, C. D. J. Am. Chem. Soc. 54 (1932) 2256.

26. Finbak, C. and Hassel, O. Z. physik. Chem. B 35 (1937) 25.

27. Fischmeister, H. F. J. Inorg. Nucl. Chem. 3 (1956) 182.

28. Schinke, H. and Sauerwald, F. Z. anorg. allgem. Chem. 304 (1960) 25.

29. Bues, W. Z. physik. Chem. (Frankfurt) 10 (1957) 1.

30. Fermor, J. H. and Kjekshus, A. Acta Chem. Scand. 24 (1970) 1015.

31. Roberts, S. Phys. Rev. 76 (1949) 1215.

32. Theimer, O. Monatsh. 81 (1950) 424.

33. Eades, R. G., Hughes, D. G. and Andrew, E. R. Proc. Phys. Soc. (London) 71 (1958) 1019.

34. Andrew, E. R., Eades, R. G., Hennel, J. W. and Hughes, D. G. Proc. Phys. Soc. (London) 79 (1962) 954.

35. Fermor, J. H. and Kjekshus, A. Acta Chem. Scand. 26 (1972) 3235.

36. Bjørseth, O., Fermor, J. H. and Kjekshus, A. Acta Chem. Scand. 25 (1971) 3791.

37. Fermor, J. H. and Kjekshus, A. Acta Chem. Scand. 21 (1967) 1265.

38. Sawada, S., Nomura, S. and Fujii, S. J. Phys. Soc. Japan 13 (1958) 1549.

39. Sawada, S., Nomura, S. and Asao, Y. J. Phys. Soc. Japan 16 (1961) 2486.

40. Khodakov, A. L. and Mirskaya, E. Z. Soviet Phys.-Cryst. (English Transl.) 7 (1962) 382.

41. Chen, A. and Chernow, F. Phys. Rev. 154 (1967) 493.

42. Leong, J. T. and Emrick, R. M. J. Phys. Chem. Solids 32 (1971) 2593.

43. Strømme, K. O. Acta Chem. Scand. 23 (1969) 1625.

Received September 22, 1972. 